29.P11

\title{
Effects of Cortical Lesions on Expression of Cell Recognition Molecules in the Corpus Striatum of Mice
}

\author{
Maciej Poltorak, Jamesine R. Williams, Antonio S. Herranz, \\ Liverana Lauretti and William J. Freed \\ NIMH Neuroscience Center at St. Elizabeths, Washington, DC 20032, USA
}

Brain injury induces trophic effects within adjacent tissue through an unknown molecular mechanism. One model of this lesion effect involves the enhanced outgrowth of neuronal processes from transplanted substantia nigra (SN) in animals with cerebral cortex lesions. Since cell recognition molecules are involved in the molecular mechanisms of contact between cells and surrounding extracellular matrix components, and are important in plasticity of the nervous system, we investigated changes in L1, $\mathrm{N}-\mathrm{CAM}$ and tenascin as well as synapse-associated proteins and gliosis in the striatum of mice with cortical lesions.

Cortical lesions were performed by aspiration of the cerebral cortex down to the corpus callosum under visual guidance. Mice were studied after $5,10,21,100$ and 150 days of survival. Brains were quickly removed, frozen, sectioned and every seventh section stained with cresyl violet. Matching frontal sections of anterior striatum from all groups were simultaneously labeled in one batch with each primary antibody using indirect immunofluorescence as the detection system. For the purpose of the study, the striatum was arbitrarily divided into five regions: the most medial-periventricular part (M), and the dorso-medial (DM), dorso-lateral (DL), ventro-lateral (VL) and ventro-medial (VM) quadrants. The medial-periventricular (M) region was defined as the area medial to the myelinated fiber bundles. Measurements of mean staining intensity were taken from both lesioned and non-lesioned sides and measured using a computer-based image analysis program.

Lesioned mice showed a significant enhancement of both L1 and N-CAM immunostaining intensity within the $\mathrm{M}$ and DM regions of the striatum, as compared to the non-lesioned side. Intensity of immunostaining with L1, NCAM and tenascin did not show significant changes related to time after lesioning. These changes in expression of both recognition molecules after frontal lesions indicate a potential predisposition of deafferented striatal tissue for neurite growth, in that the L1 and N-CAM molecules generally have promoting effects on neurite outgrowth. Our results may in part explain data suggesting that frontal cortex lesions enhance the outgrowth of neuronal processes from transplanted $\mathrm{SN}$ within the corpus striatum of rats with SN lesions (Freed and CannonSpoor, Brain Res 1988; 46: 133-143). Moreover, after frontal lesions tenascin expression was significantly decreased, but only in the $\mathrm{M}$ region of the striatum on the lesioned side. During development of the nervous system, as well as after brain injury, certain brain areas appear to express increased tenascin immunoreactivity. It has been hypothesized that this enhancement is a factor impairing neurite growth processes, as has been shown in vitro for substrate-bound tenascin. The relative lack of tenascin immunoreactivity in the $M$ region after frontal lesions may contribute to the promotion of neurite outgrowth in the medial striatum.

Lesions of the frontal cortex are assumed to have an impact on two main striatal pathways: first, they affect the corticospinal and thalamocortical projections passing through the caudateputamen, and second, they remove corticostriatal glutamate projections. Interestingly, the glutamatergic neurons that were removed project primarily to the dorsal striatur Changes seen in the present study were seen primarily in the dorso-medial region and especially in the very medial periventricular zone. The dorsolateral region was not changed. Moreover, 
GFAP and vimentin immunoreactivities were increased in almost the entire striatum on the lesioned side. For most of the striatum, gliosis was present up to 21 days after lesioning and declined thereafter. Neither vimentin nor GFAP-positive gliosis was present in the striatum on the non-lesioned side. This reactive gliosis suggests that the areas of damage were not confined only to the $\mathrm{M}$ and $\mathrm{DM}$ regions. Therefore, the areas in which cell recognition molecule expression were changed did not simply correlate with the locations of increased astrogliosis.

A decrease in synaptic protein synthesis (synapsin and synaptophysin expression) was primarily present in the medial-periventricular striatum, where it would not be expected either theoretically, from expected areas of deafferentation, or from areas of increased GFAP and vimentin staining. Any corresponding neurite damage must have been relatively small, since we did not observe significant changes in neurofilament expression. Thus, the dorsomedial region may be highly sensitive to disturbances of the corticostriatonigral circuits. This striatal area might also have a unique ability to support and promote neurite regeneration, since there is a concurrent increase in L1 and N-CAM expression and a decrease in tenascin immunoreactivity. 

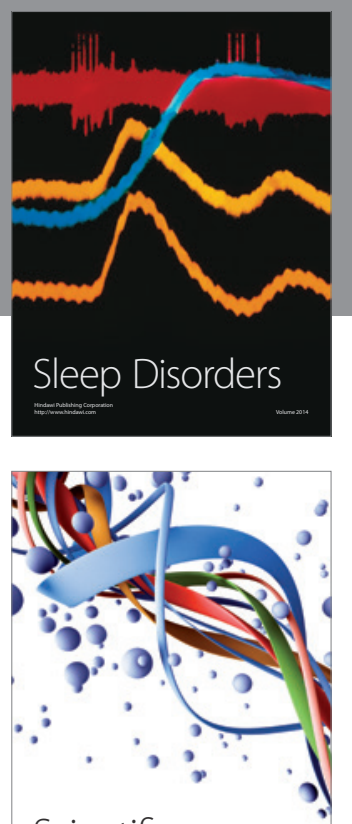

Scientifica
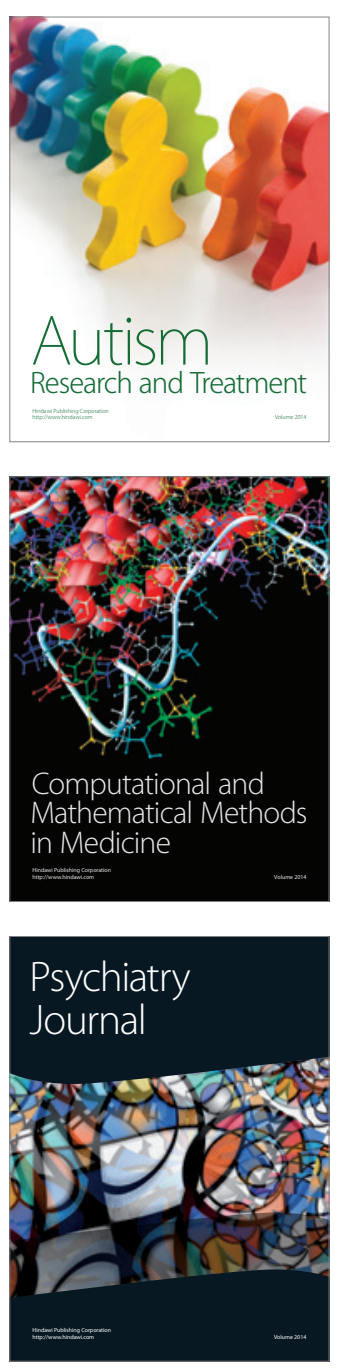
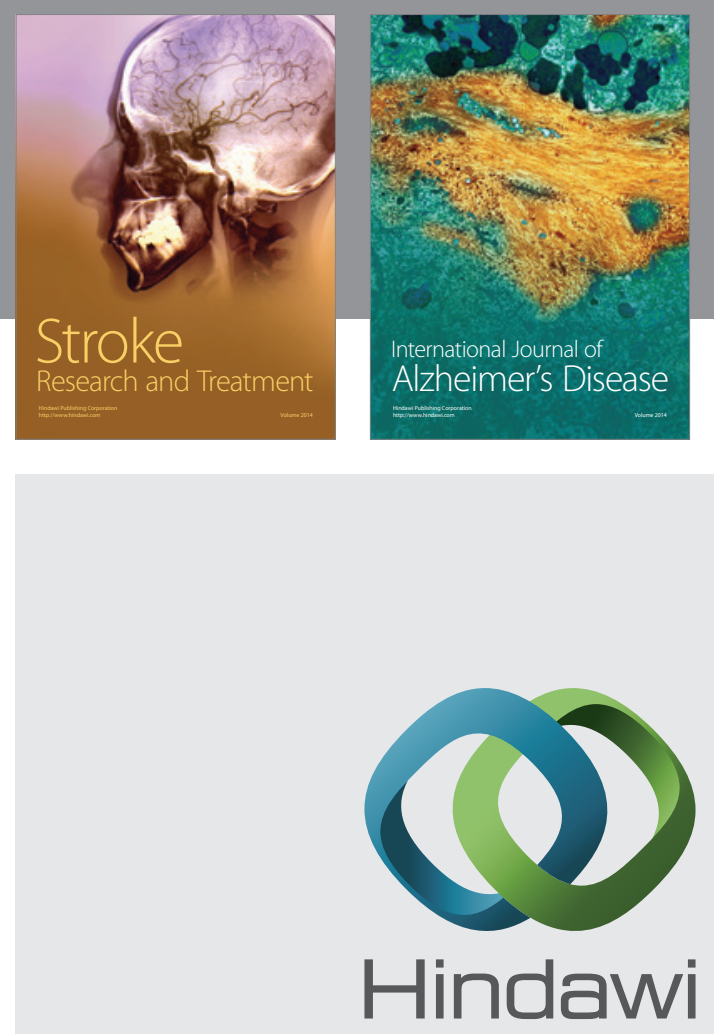

Submit your manuscripts at

http://www.hindawi.com
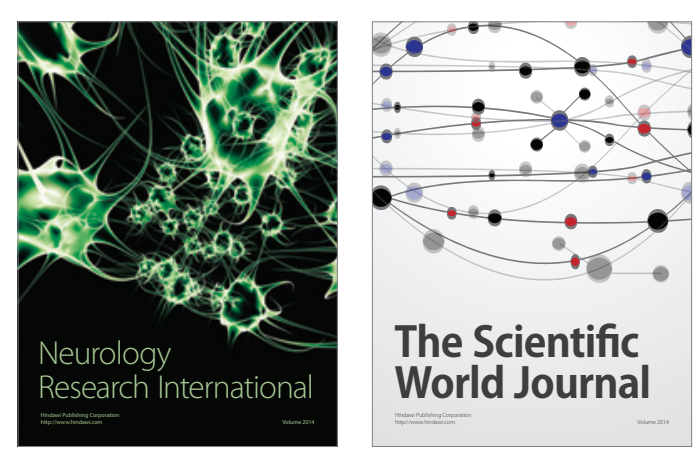

The Scientific World Journal

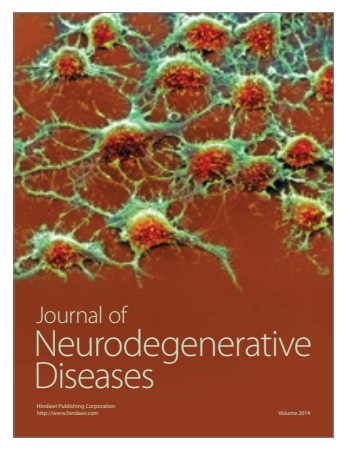

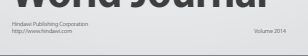

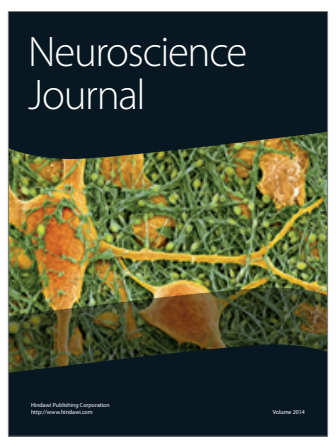

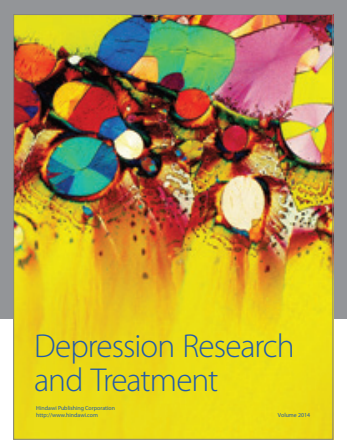
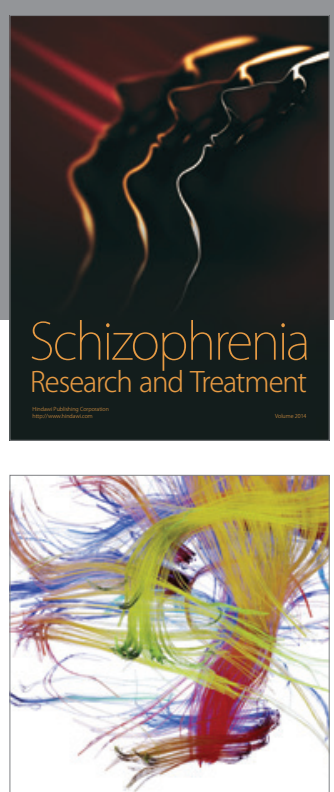

Brain Science

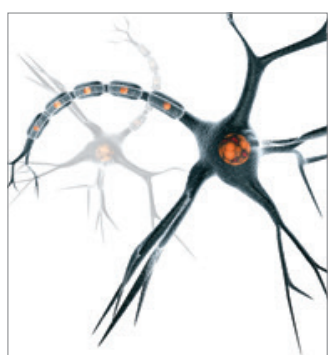

Neural Plasticity
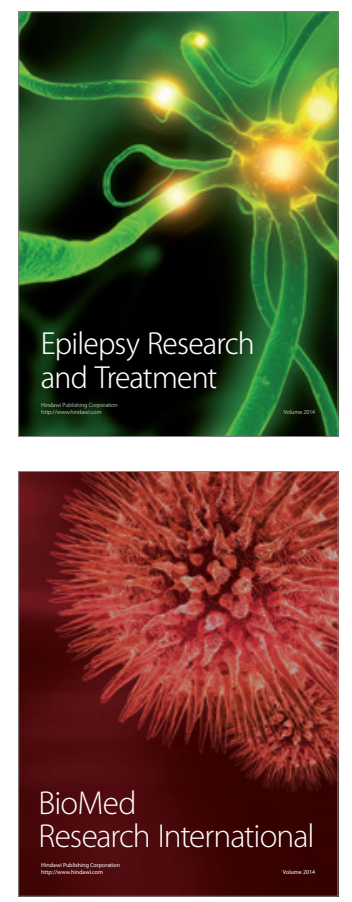

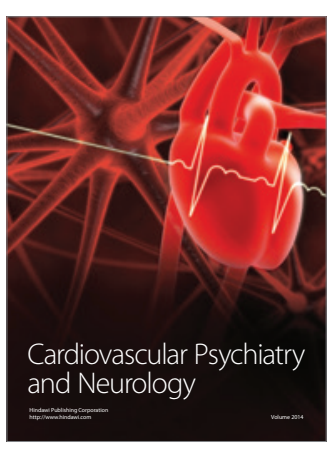

Parkinson's

Disease
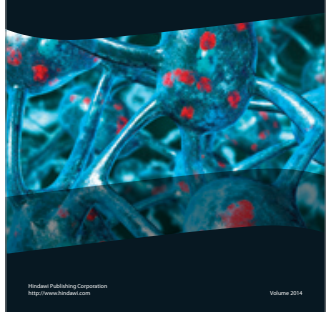